\title{
Does autoimmunity against thyroglobulin play a role in the pathogenesis of Graves' ophthalmopathy: a review
}

This article was published in the following Dove Press journal:

Clinical Ophthalmology

3 December 2015

Number of times this article has been viewed

Thayalini Shanmuganathan'

Christian Girgis ${ }^{2}$

Hooshang Lahooti'

Bernard Champion'

Jack R Wall'

'Department of Medicine, Nepean Clinical School, Nepean Hospital, The University of Sydney, Sydney,

${ }^{2}$ Department of Medicine, Westmead Millennium Institute, The University of Sydney, Sydney, NSW, Australia
Correspondence: Jack R Wall

Department of Medicine, Nepean Clinical School, Nepean Hospital, The University of Sydney, South Block, 5th Floor, Penrith, Sydney, NSW 275I, Australia

Tel +6I 247342613

Fax +6I 247342614

Email jack.wall@sydney.edu.au
Abstract: While most authors believe that autoimmunity against the TSH receptor expressed in the orbital connective tissue cells is the main reaction that leads to the development of ophthalmopathy in patients with Graves' hyperthyroidism, an older hypothesis that deserves fresh consideration is based on the notion that thyroglobulin $(\mathrm{Tg})$ in the thyroid gland passes in a retrograde fashion to the orbit where it is recognized by $\mathrm{Tg}$ autoantibodies, leading to inflammation. Here, we review new evidence that supports a role of $\mathrm{Tg}$ and propose a new hypothesis based on the notion that $\mathrm{Tg}$ is targeted in the orbit leading to a complex cascade of reactions that leads to Graves' ophthalmopathy.

Keywords: ophthalmopathy, Graves' disease, thyroglobulin, thyroid peroxidase, TSH receptor, lymphocytes, autoantibodies

\section{Introduction}

The pathogenesis of the ophthalmopathy that occurs in approximately $40 \%$ of patients with Graves' hyperthyroidism ${ }^{1}$ and, less often and usually in a less severe form, in $25 \%$ of patients with Hashimoto thyroiditis ${ }^{2}$ remains controversial. ${ }^{3,4}$ While most authors favor the hypothesis that autoimmunity against the TSH receptor (TSHr) expressed in the orbital fibroblasts, preadipocytes, and fibrocytes is the dominant autoimmune reaction that leads to orbital inflammation ${ }^{5-7}$ not all the evidence supports this, namely, Graves' disease and worsening ophthalmopathy may be associated with decreasing levels of TSHr antibodies in individual patients (Wall et al, 2013, unpublished observations) and ophthalmopathy may develop many years after the onset of hyperthyroidism. Most importantly, patients with so-called euthyroid Graves' disease ${ }^{8}$ have a similar ophthalmopathy but no evidence for thyroid autoimmunity including negative TSHr antibodies as measured in the TRAb assay and as thyroid-stimulating immunoglobulin in a Thyreporter bio-assay, ${ }^{9}$ suggesting that other possibilities should be considered. Moreover on a recent case report we showed that eye signs and other soft tissue manifestations of Graves' disease worsened in the context of psychological stress, with reduced levels of TSHr antibody, occurred following stress. ${ }^{10}$

Regardless of the nature of the inflammatory process in the orbital connective tissue and fat and extra ocular muscles (EOM), the eye disorder is presumed to begin in the thyroid since the great majority of patients with Graves' ophthalmopathy (GO) have active thyroid inflammation, ie, thyroiditis, at the time they develop eye signs. ${ }^{111}$ Thus, a logical hypothesis for the orbital reaction is that thyroid antigens are released in the context of a thyroiditis and travel ("home") to the orbital tissues where they bind to various cell types and are targeted by autoantibodies and/or sensitized 
T cells, leading to orbital inflammation. This is the working hypothesis for most groups working on ophthalmopathy and has been well studied for the TSHr, but the possible role of the other major thyroid antigen, thyroglobulin ( $\mathrm{Tg})$, has been largely ignored.

\section{The Kriss hypothesis}

An earlier hypothesis for the development of ophthalmopathy in patients with Graves' hyperthyroidism was developed by Konishi et $\mathrm{al}^{12}$ and McDougall and Kriss ${ }^{13}$ and based on the notion that $\mathrm{Tg}$ released from the thyroid gland might pass to the orbit via the putative lymphatics that link the thyroid to the orbit, where it could be recognized by Tg autoantibodies leading to orbital inflammation. Feldt-Rasmussen et al demonstrated the presence of $\mathrm{Tg}$ in the orbit but the presence of $\mathrm{Tg} / \mathrm{anti}$-Tg immune complexes has not been reported. Serum anti-Tg antibodies did not correlate closely with severity of the eye disease in one study. ${ }^{14}$

Subsequently, using a panel of well-characterized monoclonal antibodies against $\mathrm{Tg}$ and patients sera, we demonstrated reactivity against a human orbital connective tissue membrane protein which correlated with serum anti-Tg antibody levels but not ophthalmopathy. ${ }^{15} \mathrm{We}$ concluded that the orbital protein was not native Tg but another putative thyroid and orbital tissue shared antigen. In retrospect, these early studies had partly confirmed the hypothesis of McDougall and Kriss. ${ }^{13}$ Later, we carried out further studies with anti-Tg monoclonal antibodies to test for the presence of $\mathrm{Tg}$ in orbital tissues from patients with GO and, as controls, from patients undergoing orbital surgery for non-immunological disorders. However, we could not identify intact $\mathrm{Tg}$ in the orbit. ${ }^{16}$ Goh et $\mathrm{al}^{17}$ found that ophthalmopathy tended to be associated with lower titers of Tg and TPO antibodies in patients with Graves' disease compared to those without eye involvement. Although they postulated that $\mathrm{Tg}$ antibodies somehow protected against ophthalmopathy, an alternative explanation is that serum antibodies are decreased in the circulation because they are absorbed out by $\mathrm{Tg}$ in the orbital tissues.

The "Tg hypothesis" lay dormant for a further 15 years until an Italian group reported a series of experiments in which they used monoclonal antibodies against $\mathrm{Tg}$ that identified intact $\mathrm{Tg}$ in the retro-orbital tissue from three patients with GO by Western Blotting and ELISA. ${ }^{18}$ They did not find $\mathrm{Tg}$ and anti-Tg immune complexes in the orbit and concluded that autoimmunity against Tg was probably a factor in the development of ophthalmopathy but likely cell mediated. A year later the same group carried out additional studies in more patients with GO, again identifying intact $\mathrm{Tg}$ in fibroadipose tissue from three of seven patients by immunoprecipitation and Western Blotting with Tg monoclonal antibodies. They did not detect Tg in EOM extracts, similar to our own earlier findings in which eye muscle membranes did not react with our Tg monoclonal antibodies. ${ }^{19}$ They also demonstrated intact $\mathrm{Tg}$ in sub-palpebral skin extracts from two of two patients with GO and they suggested that we should re-visit the Kriss hypothesis and the possibility that $\mathrm{Tg}$ is an important target for the autoimmune reactions in ophthalmopathy. ${ }^{19}$

On the other hand, Kendall-Taylor et $\mathrm{al}^{20}$ identified an IgG which they called "ophthalmopathic immunoglobulin" that bound to retro-orbital but not to thyroid membranes, thyroid microsomes, or Tg. They concluded that GO was associated with what they called a "specific ophthalmopathic immunoglobulin" that reacted with retro-orbital antigen, as distinct from thyroid antigens, suggesting that the ophthalmopathy was an entity distinct from autoimmune thyroid disease.

\section{Serum Tg levels and ophthalmopathy}

Very recently, we demonstrated a significant positive correlation between serum $\mathrm{Tg}$ levels and the presence and severity of ophthalmopathy in patients with Graves' disease. ${ }^{21}$ In patients with Graves' disease, but not Hashimoto thyroiditis, Tg levels also correlated with serum titers of TSHr antibodies, favoring the notion that not only TSHr but also Tg, may be released from the thyroid gland in the course of a thyroiditis and home to the orbit where they become targets of autoantibodies and cytotoxic T lymphocytes.

\section{Special nature of the orbital fibroblasts}

Smith, ${ }^{22}$ Smith et al, ${ }^{23}$ Fernando et al, ${ }^{24,25}$ and Smith et $\mathrm{al}^{26}$ have convincingly demonstrated that orbital fibroblasts from patients with GO respond differently to skin fibroblasts and orbital fibroblasts from normal subjects in vitro. However, the fibroblasts may actually be the same in all situations but different in GO only because they have been primed in the context of the orbital reaction and have new surface markers which makes them seem different, ie, we need to differentiate between cause and effect before promoting the GO orbital fibroblasts, preadipocytes, and fibrocytes as "unique". However, if considered in this light, the results described by Smith et $\mathrm{al}^{23}$ are compatible with, and supportive of, the "thyroid antigen hypothesis".

While an effect of cytotoxic antibodies targeting $\mathrm{Tg}$ bound to EOM cells or orbital fibroblasts is one possible 
mechanism for the initialization of orbital inflammation, there are other potential mechanisms for reactivity against $\mathrm{Tg}$ (and other thyroid antigens) in situ in the orbit. While it is known that orbital fibroblasts can be stimulated by TSHr antibodies in vitro, ${ }^{27}$ suggesting that the prominent orbital scarring and glycosaminoglycans overproduction ${ }^{28,29}$ of GO is due to a reaction with the $\mathrm{TSHr}$, this could also result from a reaction between $\mathrm{Tg}$ antibodies and $\mathrm{Tg}$ attached to the orbital fibroblasts; more likely, both mechanisms contribute to the orbital pathology and clinical features (Table 1).

\section{Thyroiditis and ophthalmopathy}

Subacute thyroiditis ${ }^{30}$ and radioiodine treatment of Graves' hyperthyroidism may be good models for what may happen to the orbit in patients with thyroiditis. What happens to the thyroid gland following radioactive iodine treatment of patients with Graves' hyperthyroidism. Following ${ }^{131} \mathrm{I}$, there is a flare up in the thyroiditis with release of thyroid proteins and increased production of the corresponding antibodies, against $\mathrm{Tg}$, TPO, and the TSHr. ${ }^{31,32}$ In addition, we have recently shown an increase in serum $\mathrm{Tg}$ concentrations following radioiodine which correlated with an increase in $\mathrm{TSHr}$ antibody titer, initial worsening of the eye signs, but later improvement (Wall et al, 2013, unpublished observations). It is also likely, but not yet shown, that the numbers of thyroid antigen-sensitized $\mathrm{T}$ cells increase in the thyroid following ${ }^{131}$ I treatment; antibodies and sensitized T cells could pass to the orbital tissues, leading to a flare up in the severity of existing eye disease. Indeed, studies confirming that radioactive iodine treatment leads to worsening of eye signs - or even the new development of ophthalmopathy, although this is more controversial - would be good evidence for a role of autoimmunity against thyroid antigens in the development of ophthalmopathy in patients with thyroid autoimmunity. ${ }^{33,34}$ While there is no mechanistic data that radioactive iodine worsens $\mathrm{GO}$ by increasing thyroid destruction, release of $\mathrm{Tg}$, and production of Tg/anti-Tg immune complex, this is our working hypothesis for present in vitro and clinical studies (Table 2). In the longer term, one would expect a decrease in the severity of the ophthalmopathy as the "thyroid antigen mass" is destroyed by ${ }^{131} \mathrm{I}$, and this has been shown in earlier observations that total thyroidectomy is associated with the improvement of existing eye disease and prevention of new ophthalmopathy. ${ }^{35-37}$

While this review focuses on the role of $\mathrm{Tg}$, we believe that there are other thyroid antigens that are involved in the pathogenic process of GO. This process can be characterized as a complex series of reactions involving multiple thyroid antigens, the corresponding serum autoantibodies, cellular immunity, cytokines, and a large number of other chemical mediators and co-factors. Our proposed hypothesis for the role of $\mathrm{Tg}$ in the development of ophthalmopathy in patients with thyroid autoimmunity is shown schematically in Figure 1.

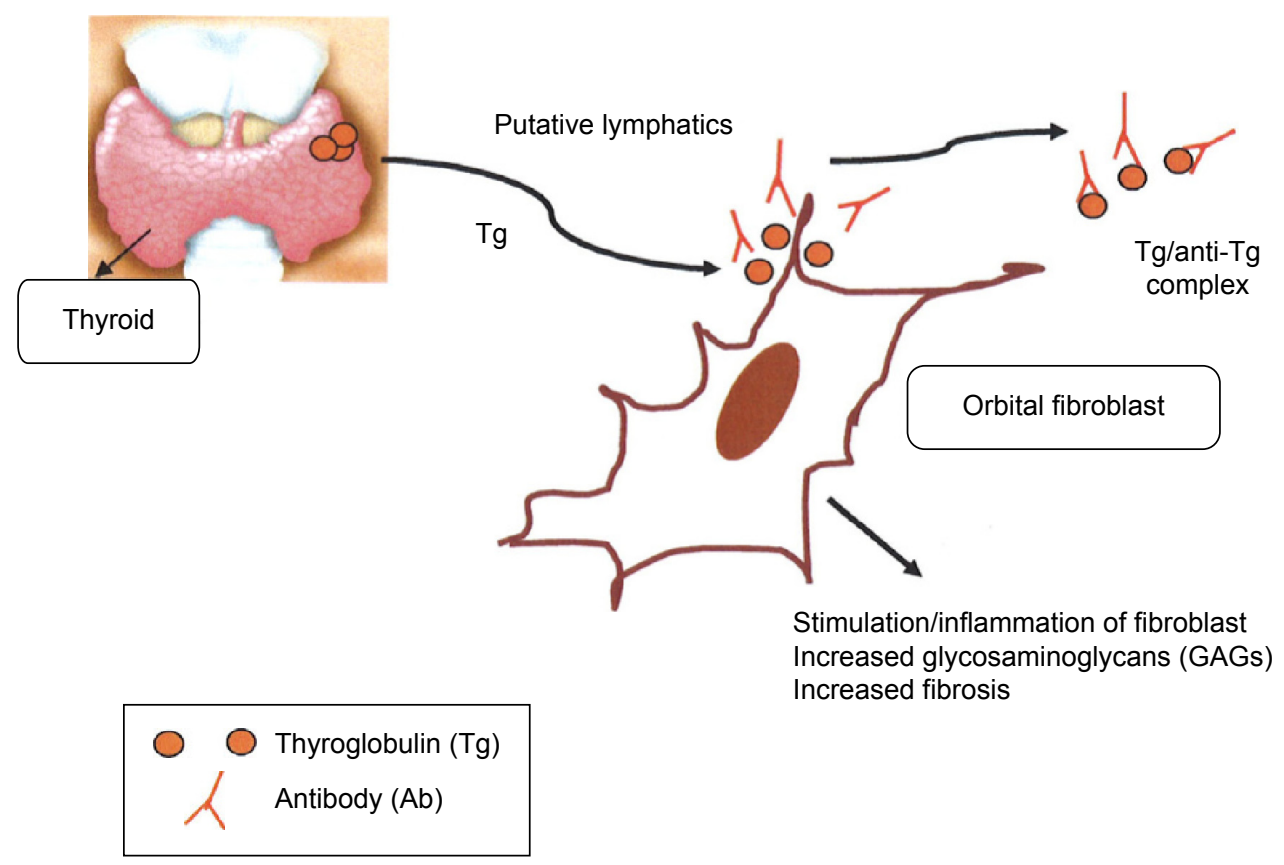

Figure I Hypothetical role of thyroglobulin in Graves' ophthalmopathy. 
Table I Evidence supporting a role of thyroglobulin in the pathogenesis of Graves' ophthalmopathy

I. Thyroglobulin is a large and very antigenic protein and the corresponding autoantibodies react with it in many clinical and experimental situations, so why not in the orbit?

2. Intact thyroglobulin can be detected in the orbits of patients with Graves' ophthalmopathy so would be seen by circulating thyroglobulin antibodies

3. In patients with Graves' hyperthyroidism, serum thyroglobulin levels correlate with the prevalence and severity of any associated ophthalmopathy

4. In patients with Graves' ophthalmopathy, serum thyroglobulin levels correlate with serum titers of TSHr antibodies

5. In patients with Graves' ophthalmopathy, serum thyroglobulin levels correlate with TSHr antibody titers, suggesting that both thyroid antigens are released at the same time

6. Thyroglobulin antibodies in the circulation and from the thyroid would bind to $\mathrm{Tg}$ in the orbit, although this has not been demonstrated

Abbreviation: $\mathrm{TSHr}$, TSH receptor.

\section{Role of chemokines in GO}

The importance of chemokines such as CXCL10 and CCL2 on GO has been investigated by the Italian team. ${ }^{38,39}$ In their in vivo and in vitro studies, they have demonstrated that EOM involves in the self-perpetuation of inflammation by secreting CXCL10 and CCL2 chemokines under the influence of cytokines especially IFN $\gamma$. In addition to that, CXCL9 and CXCL10 have been assessed as surrogate markers of GO while patients undergoing treatment and were proposed to be useful guidelines in therapeutic decision-making. ${ }^{40}$

\section{Future studies}

The evidence for a role of autoimmunity against $\mathrm{Tg}$ is, as is the case for the TSHr, mainly circumstantial. In order to prove the "thyroid antigen hypothesis", we must demonstrate that normal orbital fibroblasts and EOM cells, to which are attached $\mathrm{Tg}$, become targets for antibodies and/or sensitized $\mathrm{T}$ lymphocytes from patients with ophthalmopathy, but not from those without eye disease. We can compare GO patient sera vs normal sera, fibroblasts to which is bound $\mathrm{Tg}$ vs control antigen and peripheral blood mononuclear cells, or

Table 2 Proposed pathogenic mechanisms of Tg for Graves' ophthalmopathy

I. Thyroglobulin antibodies bind to native thyroglobulin which is attached to the orbital fibroblasts. Subsequently the production of thyroglobulin/anti-thyroglobulin immune complexes stimulate the fibroblast, contributing to the orbital inflammation.

2. CD8 ${ }^{+}$cytotoxic $T$ lymphocytes directed against the extra ocular muscle fiber are also likely to be involved, but specific targets and effects of the reactions are not understood purified T-cell subsets from patients with GO vs those from patients with Graves' disease without eye signs. We can also isolate mononuclear cells from the thyroid of patients with GO and test for reactivity against the same target cells vs mononuclear cells from patients with Graves' disease without ophthalmopathy. Specific IgG autoantibodies obtained by affinity chromatography, and purified subpopulations of $\mathrm{T}$ cells targeting $\mathrm{Tg}$ can be incubated with Tg-coated EOM cells or orbital fibroblasts to confirm findings using serum and unfractionated mononuclear cells. As end points, one can measure changes in gene expression for inflammatory markers and cytokine levels. It is also possible to study the putative benefits of selenium supplementation in GO in this model. We can carry out serial studies of patients with Graves' hyperthyroidism, measuring serum $\mathrm{Tg}$ antibodies and correlating with eye signs. The notion that $\mathrm{Tg}$ antibodies tend to be low in patients with ophthalmopathy and those without eye disease due to their absorption by the orbital tissues can be studied by correlating orbital Tg levels in immunohistochemistry with parameters of the orbital autoimmune reactions, scores for eye signs, and serum $\mathrm{Tg}$ and anti-Tg titers in serial studies of patients with and without ophthalmopathy, including those treated with radioactive iodine.

\section{Conclusion}

The orbital reactions in patients with Graves' disease are complex, involving antibodies and $\mathrm{T}$ lymphocytes, as well as other mononuclear cells, homing receptors on orbital cells, and cytokines. Moreover, we must determine why it is that only approximately $50 \%$ of patients with Graves' hyperthyroidism develop ophthalmopathy. Environmental and genetic factors will play a role, an example being the presence of a recently shown informative single nucleotide polymorphism in the CASQ1 gene which is linked to ophthalmopathy. ${ }^{41}$ Smoking is well recognized as a major risk factor for ophthalmopathy and stress is likely to play an important role in its initiation. ${ }^{42}$ In conclusion, it does seem appropriate to give serious attention to the possibility that the pathogenesis of $\mathrm{GO}$ is not just about the TSHr but the contribution from $\mathrm{Tg}$ and other thyroid antigens as well.

\section{Disclosure}

The authors report no conflicts of interest in this work.

\section{References}

1. Wiersinga WM, Bartalena L. Epidemiology and prevention of Graves' ophthalmopathy. Thyroid. 2002;12:855-860.

2. Tjiang H, Lahooti H, McCorquodale T, Parmar KR, Wall JR. Eye and eyelid abnormalities are common in patients with Hashimoto's thyroiditis. Thyroid. 2010;20:287-290. 
3. Wall J. Thyroid function: news and views: pathogenesis of Graves' ophthalmopathy - a role for TSH-R? Nat Rev Endocrinol. 2014;37: 256-258.

4. Girgis CM, Champion BL, Wall JR, Girgis C, Champion B, Wall J. Current concepts of Graves' disease: a review. Ther Adv Endocrinol Metab. 2012; 2:135-144.

5. Weetman AP. Graves' disease. N Engl J Med. 2000;343:1236-1248.

6. Eckstein AK, Plicht M, Lax H, et al. Thyrotropin receptor autoantibodies are independent risk factors for Graves' ophthalmopathy and help to predict severity and outcome of the disease. J Clin Endocrinol Metab. 2006;91:3464-3470.

7. Paschke R, Vassart G, Ludgate M. Current evidence for and against the TSH receptor being the common antigen in Graves' disease and thyroid associated ophthalmopathy. Clin Endocrinol (Oxf). 1995;42:565-569.

8. McCorquodale T, Lahooti H, Gopinath B, Wall JR. Long term follow-up of seven patients with ophthalmopathy not associated with thyroid autoimmunity - heterogeneity of autoimmune ophthalmopathy. Clin Ophthalmol. 2012;6:1-9.

9. Lahooti H, Houtz JA, Tran YC, et al. TSH receptor antibodies as measured in a thyroid stimulating immunoglobulin (TSI) reporter bioassay are not detected in patients with euthyroid Graves' disease. Austin J Clin Ophthalmol. 2014;1:1-7.

10. Girgis CM, Champion BL, Wall JR. Psychological stress and soft-tissue manifestations of Graves' disease: a potential link? AACE Clin Case Rep. In press 2015.

11. Bartley GB, Fatourechi V, Kadrmas EF, et al. Clinical features of Graves' ophthalmopathy in an incidence cohort. Am J Ophthalmol. 1996; 121:284-290.

12. Konishi J, Herman MN, Kriss JP. Binding of thyroglobulin and thyroglobulin-anti thyroglobulin complex to extraocular muscle membrane. Endocrinology. 1974;95:434-446.

13. McDougall IR, Kriss JP. New thoughts about the cause and treatment of the severe ocular manifestations of Graves' disease. Scot Med J. 1974;19:165-169.

14. Feldt-Rasmussen U, Kemp A, Bech K, Madsen AN, Date J. Serum thyroglobulin, its autoantibody and thyroid stimulating antibodies in the endocrine exophthalmos Acta Endocrinol (Coph). 1981;96:192-198.

15. Kuroki T, Ruf T, Whelan L, Miller A, Wall JR. Antithyroglobulin monoclonal and autoantibodies cross-reactivity on orbital connective tissue antigen. A possible mechanism for the association of ophthalmopathy with autoimmune thyroid disorders. Clin Exp Immunol. 1985;69:931-936.

16. Kodama K, Sikorska H, Bayly R, Bandy-Dafoe P, Wall J. Use of monoclonal antibodies to investigate a possible role of thyroglobulin in the pathogenesis of Graves' ophthalmopathy. J Clin Endocrinol Metab. 1974;59:67-73.

17. Goh SY, Ho SC, Seah LL, Fong KS, Khoo DH. Thyroid autoantibody profiles in ophthalmic dominant and thyroid dominant Graves' disease differ and suggest ophthalmopathy is a multi antigenic disease. Clin Endocrinol (Oxf). 2004;60:600-607.

18. Marino M, Lisi S, Pinchera A, et al. Identification of thyroglobulin in orbital tissues of patients with thyroid-associated ophthalmopathy. Thyroid. 2001;11:177-185.

19. Marinò M, Chiovato L, Lisi S, et al. Role of thyroglobulin in the pathogenesis of Graves' ophthalmopathy: the hypothesis of Kriss revisited. J Endocrinol Invest. 2004;27:230-236.

20. Kendall-Taylor P, Atkinson S, Holcombe M. Specific IgG in Graves' ophthalmopathy and its relation to retro-orbital and thyroid autoimmunity. BMJ. 1984;288:1183-1186.

21. Lahooti H, Shanmuganathan T, Champion B, Wall JR. Serum thyroglobulin levels in patients with thyroid autoimmunity with and without ophthalmopathy or isolated upper eyelid retraction. Int Trends Immun. 2015;3:22-27.

22. Smith TJ. TSH-receptor-expressing fibrocytes and thyroid associated ophthalmopathy. Nat Rev Endocrinol. 2015;11:171-181.
23. Smith TJ, Hegedüs L, Douglas RS. Role of insulin-like growth factor-1 (IGF-1) pathway in the pathogenesis of Graves' orbitopathy. Best Pract Res Clin Endocrinol Metab. 2012;26:291-302.

24. Fernando R, Lu Y, Atkins SJ, Mester T, Branham K, Smith TJ. Expression of thyrotropin receptor, thyroglobulin, sodium-iodide symporter and thyroperoxidase by fibrocytes depends on AIRE. Clin Endocrinol Metab. 2014;99:E1236-E244.

25. Fernando R, Atkins S, Raychaudhuri N, et al. Human fibrocytes coexpress thyroglobulin and thyrotropin receptor. Proc Natl Acad Sci US A. 2012;109:7427-7432.

26. Smith TJ, Tsa C-C, Shih M-J, et al. Unique attributes of orbital fibroblasts and global alterations in IGF-1 receptor signaling could explain thyroid-associated ophthalmopathy. Thyroid. 2008;18:983-988.

27. Rotella CM, Zonefrati R, Toccafondi R, Valente WA, Kohn LD. Ability of monoclonal antibodies to the thyrotropin receptor to increase collagen synthesis in human fibroblasts: an assay which appears to measure exophthalmogenic immunoglobulins in Graves' sera. J Clin Endocrinol Metab. 1986;62:357-367.

28. Hansen C, Fraiture B, Rouhi R, Otto E, Förster G, Kahaly G. HPLC glycosaminoglycan analysis in patients with Graves' disease. Clin Sci (Lond). 1997;92:511-517.

29. Kahaly G, Stover C, Otto E, Beyer J, Schuler M. Glycosaminoglycans in thyroid-associated ophthalmopathy. Autoimmunity. 1992;13:81-88.

30. Madeddu G, Casu AR, Costanza C, et al. Serum thyroglobulin levels in the diagnosis and follow-up of subacute 'painful' thyroiditis. A sequential study. Arch Intern Med. 1985;145:243-247.

31. Feldt-Rasmussen U, Bech K, Date J, et al. Thyroid stimulating antibodies, thyroglobulin antibodies and serum proteins during treatment of Graves' disease with radioiodine or propylthiouracil. Allergy. 1982;37:161-167.

32. Einhorn J, Fagraeus A, Jonsson J. Thyroid antibodies after 131I treatment for hyperthyroidism. J Clin Endo Metab. 1965;25:1218-1224.

33. Bartelena L, Marocci C, Bogazzi F, Panicucci M, Lepri A, Pinchera A. Use of corticosteroids to prevent progression of Graves' ophthalmopathy after radioiodine therapy for hyperthyroidism. $N$ Engl $J$ Med. 1989;321:1349-1352.

34. Marcocci C, Bartalena L, Bogazzi F, Bruno-Bossio G, Pinchera A. Relationship between Graves' ophthalmopathy and type of treatment of Graves' hyperthyroidism. Thyroid. 1992;2:171-178.

35. Boyle IT, Greig WR, Thomson JA, Winning J, McGirr EM. Effect of thyroid ablation on dysthyroid exophthalmos. Proc R Soc Med. 1969;62(1):19-23.

36. Catz B, Perzik SL. Total thyroidectomy in the management of thyrotoxic and euthyroid Graves' disease. Am J Surg. 1969;118:434-439.

37. Kurihara $\mathrm{H}$. Total Thyroidectomy for the treatment of hyperthyroidism in patients with ophthalmopathy. Thyroid. 2002;12:265-267.

38. Antonelli A, Ferrari SM, Corrado A, et al. Extra-ocularmuscle cells from patients with Graves' ophthalmopathy secrete $\alpha$ (CXCL10) and $\beta$ (CCL2) chemokines under the influence of cytokines that are modulated by PPAR $\gamma$. Autoimmun Rev. 2014;13:1160-1166.

39. Antonelli A, Rotondi M, Ferrari SM, et al. Interferon- $\gamma$-inducible $\alpha$-chemokine CXCL10 involvement in Graves' ophthgalmopathy: modulation by peroxisome proliferator-activated receptor- $\gamma$ agonists. J Clin Endocrinol Metab. 2006;91:614-620.

40. Mysliwiec J, Palyga I, Kosciuszko M, Kowalska A, Gorska M. Circulating CXCL9 and CXCL10 as markers of activity of Graves' orbitopathy during treatment with corticosteroids and teleradiotherapy. Horm Metab Res. 2012;44(13):957-961.

41. Lahooti H, Cultrone D, Edirimanne S, et al. Association of the CASQ1 gene rs3838216 with Graves' ophthalmopathy and Hashimoto's thyroiditis in patients with thyroid autoimmunity. Ophthalmol Res. 2014;2: 281-229.

42. Mizokami T, Li AW, Wall JR. Stress and thyroid autoimmunity. Thyroid. 2004;14:1047-1055. 


\section{Publish your work in this journal}

Clinical Ophthalmology is an international, peer-reviewed journal covering all subspecialties within ophthalmology. Key topics include: Optometry; Visual science; Pharmacology and drug therapy in eye diseases; Basic Sciences; Primary and Secondary eye care; Patient Safety and Quality of Care Improvements. This journal is indexed on

PubMed Central and CAS, and is the official journal of The Society of Clinical Ophthalmology (SCO). The manuscript management system is completely online and includes a very quick and fair peer-review system, which is all easy to use. Visit http://www.dovepress.com/ testimonials.php to read real quotes from published authors. 